\title{
Efficacy and Safety of Ferric Carboxymaltose and Other Formulations in Iron-Deficient Patients: A Systematic Review and Network Meta-analysis of Randomised Controlled Trials
}

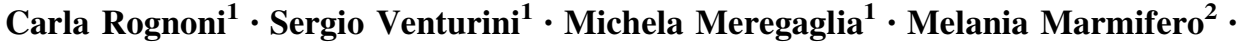 \\ Rosanna Tarricone $\mathrm{e}^{1,3}$
}

Published online: 21 December 2015

(c) The Author(s) 2015. This article is published with open access at Springerlink.com

\begin{abstract}
Background Iron deficiency is very common in a number of medical conditions. Ferric carboxymaltose is a new stable iron preparation that can be administered in single infusions over short periods of time. The aim of this study was to conduct a systematic review of randomised controlled trials (RCTs) regarding the efficacy and safety of the novel complex compared with other iron formulations. In addition, the feasibility of a network meta-analysis for indirect comparisons was investigated.

Methods A systematic literature review was performed for published RCTs on the use of ferric carboxymaltose in iron deficiency between July and October 2014. Indirect comparisons were also addressed using terms referring to competing iron formulations. We further supported the qualitative results of the systematic review by a network meta-analysis that allows pooling the evidence around different intervention outcomes in the absence of trials involving a direct comparison.

Results The initial search yielded 1027 citations, which was decreased to 21 studies eligible for inclusion in the review. Studies were heterogeneous in the number of patients randomised, iron deficiency-related conditions addressed, trial inclusion criteria, time horizon, treatment dosage and outcomes assessed. Six studies with the same time horizon (i.e. 6 weeks) were included in the network meta-analysis.
\end{abstract}

Carla Rognoni

carla.rognoni@unibocconi.it

1 Centre for Research on Health and Social Care Management (CERGAS), Bocconi University, Via Roentgen 1, 20136 Milan, Italy

2 Transfusion Medicine, San Lazzaro Hospital, Alba, Italy

3 Department of Policy Analysis and Public Management, Bocconi University, Via Roentgen 1, Milan, Italy
Considering the differences between final and initial outcome values for each iron formulation, the mean difference of these differences (delta) was estimated for each couple of treatments involving ferric carboxymaltose. Significant improvements in serum ferritin $(\mu \mathrm{g} / \mathrm{l})$ were obtained with ferric carboxymaltose compared to oral iron (delta $172.8 ; 95 \% \mathrm{CI}$ 66.7-234.4) and in haemoglobin $(\mathrm{g} / \mathrm{dl})$ with respect to ferric gluconate (delta 0.6 ; $95 \%$ CI $0.2-0.9$ ), oral iron (delta 0.8 ; 95 \% CI 0.6-0.9) and placebo (delta 2.1; $95 \%$ CI 1.2-3.0).

Conclusions All currently available intravenous iron preparations appear to be safe and effective, but ferric carboxymaltose seems to provide a better and quicker correction of haemoglobin and serum ferritin levels in irondeficient patients.

\section{Key Points}

Ferric carboxymaltose is a new intravenous iron formulation that allows the administration of high doses of iron over a limited time. Although it was proved to be effective and safe in different clinical studies, extensive use in iron-deficient patients was not supported by strong scientific evidence.

The aim of this study was to conduct a systematic review and network meta-analysis of randomised controlled trials to combine the highest quality (direct and indirect) evidence regarding the efficacy and safety of ferric carboxymaltose compared with other available iron formulations.

Ferric carboxymaltose seems to provide a better and more rapid correction of haemoglobin and serum ferritin levels in iron-deficient patients compared to other iron formulations. 


\section{Introduction}

Iron deficiency (ID) is the most common nutritional disorder in the world affecting most preschool children and pregnant women in developing countries and at least 30-40\% in industrialised countries [1,2]. This condition also occurs frequently across multiple therapeutic areas and especially in patients with chronic disease (such as inflammatory bowel disease and chronic kidney disease). ID can result from chronic blood loss, decreased dietary intake, reduced intestinal absorption, or impaired use of endogenous iron due to chronic inflammations [3]. Common symptoms that may result from ID are fatigue, susceptibility to stress, lack of concentration and underperformance. ID is also associated with increased risk of infections [3], besides representing the most common cause of anaemia worldwide. In particular, the WHO defines anaemia as haemoglobin $(\mathrm{Hb})<12 \mathrm{~g} / \mathrm{dl}$ in non-pregnant women and $<13 \mathrm{~g} / \mathrm{dl}$ in men [2]. In developed countries, iron deficiency anaemia (IDA) occurs in $2-5 \%$ of adult men and postmenopausal women and represents a common cause of hospitalisation, morbidity and quality-oflife impairment [4].

Treatment for anaemic patients should involve prompt iron replacement plus diagnostic steps directed towards identifying the underlying cause of IDA [5]. Non-anaemic subjects with low serum ferritin concentration with symptoms of fatigue may also benefit from iron therapies [6]. Oral iron supplementation is usually the first treatment choice for iron repletion; however, intravenous iron may be better suited to those patients who are unable to tolerate oral iron intake due to gastrointestinal side effects or whose chronic iron loss exceeds the replacement rate achievable with oral therapy [7]. Parenteral iron formulations are also prescribed when there is a need for rapid delivery of iron as in pregnancy or following traumas [7-9] and in any situations where blood transfusions should be avoided [10, 11].

The first iron intravenous preparations were associated with acute toxicity deriving from the release of free iron. Nowadays, all parenteral therapies are formulated so that each iron particle is surrounded by a carbohydrate molecule which allows a slow release of iron and limits toxicity. Current intravenous iron formulations include high or low molecular weight iron dextran, ferric gluconate, iron sucrose and, very recently, ferric carboxymaltose [12]. They all share the same structure, but differ from each other by the size of the core and the identity and density of the surrounding carbohydrate.

Ferric carboxymaltose is an innovative, intravenous iron preparation surrounded by a non-dextran carbohydrate shell-the stable ferric carboxymaltose complex, which allows the release of iron in a controlled manner. Thanks to this intrinsic property, ferric carboxymaltose can be administered in a short period of time (15 min) and at large doses (up to $750 \mathrm{mg}$ in the USA and $1000 \mathrm{mg}$ in EU) ensuring the amount of iron needed to promptly relieve patients from the debilitating effects of ID [13]. Ferric gluconate was approved in 2002 for use in patients undergoing haemodialysis and rapidly replaced iron dextran due to severe adverse events (AEs; i.e. anaphylaxis reactions) associated with the latter formulation; its recommended dose for adult patients is $125 \mathrm{mg}$ per treatment (from the leaflet [14]). Iron sucrose represents another intravenous alternative to treat haemodialysis-related IDA; iron sucrose can be safely administered as a bolus infusion over $2 \mathrm{~min}$ or as a short infusion for doses up to $300 \mathrm{mg}$. A last option, ferumoxytol, is available to treat adult patients with IDA associated with chronic kidney disease in the USA; however, since it may cause serious hypersensitivity reactions (including death), from 2014 this drug is no longer authorised in Europe.

In synthesis, current treatments with intravenous iron either risk anaphylaxis when using iron dextran or require multiple injections of low doses when using non-dextrancontaining agents (i.e. ferric gluconate and iron sucrose). Although ferric carboxymaltose appears as an attractive option in terms of both efficacy and safety, a widespread use of this formulation is not yet supported by a high level of evidence. Moore and colleagues [15] examined the available trials of intravenous ferric carboxymaltose using details from both published and unpublished literature. The study increased the scientific evidence supporting recommendations for intravenous iron treatments - and for ferric carboxymaltose in particular-versus oral iron, but also highlighted the paucity of trial data comparing different parenteral iron preparations [15].

The aim of this study was to conduct a systematic review and perform a network meta-analysis (NMA) of randomised controlled trials (RCTs) to combine the highest quality evidence regarding the efficacy and safety of the novel iron complex (ferric carboxymaltose) compared (directly or indirectly) to other existing (intravenous and oral) formulations.

\section{Methods}

The present review was conducted in keeping with the current guidelines from the Preferred Reporting Items for Systematic Reviews and Meta-Analyses (PRISMA) [16]. All reviewing activities were conducted by two independent reviewers (CR and $\mathrm{MM}$ ) with disagreements resolved by consensus. 


\subsection{Literature Search}

Literature searches were performed for published RCTs on the use of intravenous ferric carboxymaltose for the treatment of ID in any medical condition. Published studies were identified by searching PubMed, MEDLINE, EMBASE and The Cochrane Library using "ferric carboxymaltose" OR "Ferinject" OR "Injectafer" as keywords in the title, abstract or anywhere in a document. In order to perform (direct or indirect) comparisons of ferric carboxymaltose with other preparations, terms referring to competing iron formulations (i.e. "Ferlixit" OR "ferric gluconate"; "Venofer" OR "iron sucrose"; "oral iron" OR "ferrous sulphate") were included in the query. Due to current restrictions involving the prescription of ferumoxytol, this intravenous iron formulation was not considered in the analysis. The search was limited to RCTs using highly sensitive filters; electronic searches were conducted originally between July and October 2014. No language or journal restriction was enforced in order to minimise the risk of publication bias.

Studies combining the administration of iron and erythropoiesis-stimulating agents were excluded from the analysis in order to evaluate the effect of iron treatment alone. Moreover, trials addressing anaemia following surgical interventions or studies not reporting the haematopoietic response among the outcomes were not considered.

\subsection{Data Extraction}

The following data were extracted from each study: first author's last name, publication year, title, study horizon, number of participants (per arm), ID-related medical condition, intervention and comparator, drug dosage, post-intervention efficacy [i.e. $\mathrm{Hb}$, serum ferritin, transferring saturation (TSAT)] and safety (i.e. number and type of AEs) outcome values.

\subsection{Quality Assessment}

The evaluation of potential biases in the selected studies is an essential element of a systematic literature review or meta-analysis. The internal validity of the eligible studies was assessed according to the Cochrane Collaboration's Risk of Bias tool in Review Manager (RevMan 5-http:// tech.cochrane.org/revman). The risk of bias assessment was performed with reference to the following domains: sequence generation; allocation concealment; blinding of participants and personnel and outcome assessors; blinding of outcome assessment; incomplete outcome data; selective outcome reporting.

\subsection{Outcome Measures}

The primary efficacy endpoint to be evaluated in the included studies was the haematopoietic response, usually defined as the improvement in $\mathrm{Hb}$ levels $(\mathrm{g} / \mathrm{dl})$ achieved by the two (or more) iron formulations compared. Secondary outcomes were the proportion of patients achieving correction (or avoiding a recurrence) of IDA, time to reach the target haematopoietic response $(\mathrm{Hb} \geq 12 \mathrm{~g} / \mathrm{dl})$, increase in TSAT (percentage) and serum ferritin $(\mathrm{ng} / \mathrm{ml})$, and improvement in symptoms of ID-related diseases. Safety outcomes as the proportion of study participants reporting serious or mild treatment-related AEs were also included.

Categorical variables were described as absolute (and percentage) frequencies and continuous variables as mean ( \pm standard deviation).

\subsection{Network Meta-analysis}

In order to quantitatively support the findings of the systematic review, a network meta-analysis (NMA) was conducted using data extracted from the RCTs identified through the same literature search.

Systematic reviews of RCTs are considered the standard basis in evidence-based medicine to inform clinical treatment guidelines and reimbursement policies. Many systematic reviews use meta-analysis to combine quantitative results of comparable studies and summarise the available evidence [17]. In the absence of trials involving a direct comparison of all the treatments of interest, an indirect comparison can represent an effective alternative to generate enough evidence to select the best treatment option [18]. NMA is a generalization of standard pairwise metaanalysis that allows pooling both the direct and the indirect evidence available for a given intervention. In this way, it is possible to obtain a more precise estimate of the relative effect for each pair of treatments considered $[19,20]$.

In the past few years, NMAs have been increasingly adopted for comparing healthcare interventions [21-23] in different therapeutic areas and, subsequently, endorsed by several health-technology assessment bodies [e.g. Canadian Agency for Drugs and Technologies in Health, National Institute for Health and Clinical Excellence (NICE; UK)].

A Bayesian NMA implemented by using either fixed or random effects [24] has been carried out in WinBUGS [25]. The choice between the two models has been addressed by comparing the associated information criterion [i.e. the Deviance Information Criterion (DIC)], where the lower the value, the better the fit of the model to the data. 


\section{Results}

\subsection{Study Selection}

The initial search from multiple databases yielded 1027 citations, decreasing to 400 after removal of duplicates. A subsequent review of the abstracts yielded 129 articles that were evaluated in depth; eventually, 21 studies were eligible for inclusion in our systematic review (Fig. 1).

\subsection{Study Characteristics}

The characteristics of the 21 studies included in the systematic review are summarised in Table 1. All were RCTs comparing iron treatments in anaemic (or nonanaemic) patients requiring therapies for ID. Studies were published between 2003 and 2014. The time horizon of each trial spanned between 2 weeks $[8,26]$ and 9 months [27]. The number of patients enrolled ranged between 18 [26] and 2584 [28], averaging at 472 subjects $( \pm 703)$. The vast majority of studies $(n=16)$ [8, 9, 12, 28-39] recruited adult patients ( $\geq 18$ years) with anaemia (i.e. $\mathrm{Hb}$ $<13 \mathrm{~g} / \mathrm{dl}$ in men and $<12 \mathrm{~g} / \mathrm{dl}$ in women), although the haematological inclusion criteria varied among studies. Indeed, in some articles the target population included patients with severe IDA (i.e. $\mathrm{Hb}<10.5 \mathrm{~g} / \mathrm{dl}$ ) only [7, 9, 32, 38], while in others [33] these severe cases were explicitly excluded from the analysis. Two studies enrolled premenopausal non-anaemic women with symptoms of fatigue and low ferritin levels $(\leq 50 \mathrm{ng} / \mathrm{ml})[6$, 40], while two others [26, 41] addressed a neurological disorder (i.e. the restless legs syndrome) which may be caused or worsened by ID. Finally, one study [42] evaluated IDA recurrence in patients with inflammatory bowel disease whose $\mathrm{Hb}$ levels where above IDA upper limits at the time of study entry.

IDA patients across the studies were affected by a variety of conditions including inflammatory bowel disease (and related thrombocytosis), chronic kidney disease, nonvariceal acute upper gastrointestinal bleeding, chronic heart failure and post-partum uterine bleeding. Other participants experienced bleeding owing to invasive techniques such as apheresis and haemodialysis.

\section{7 recordsidentified through database searching: \\ EMBASE:262, PubMed:256, Ovid MEDLINE: 229 The Cochrane Library: 280}

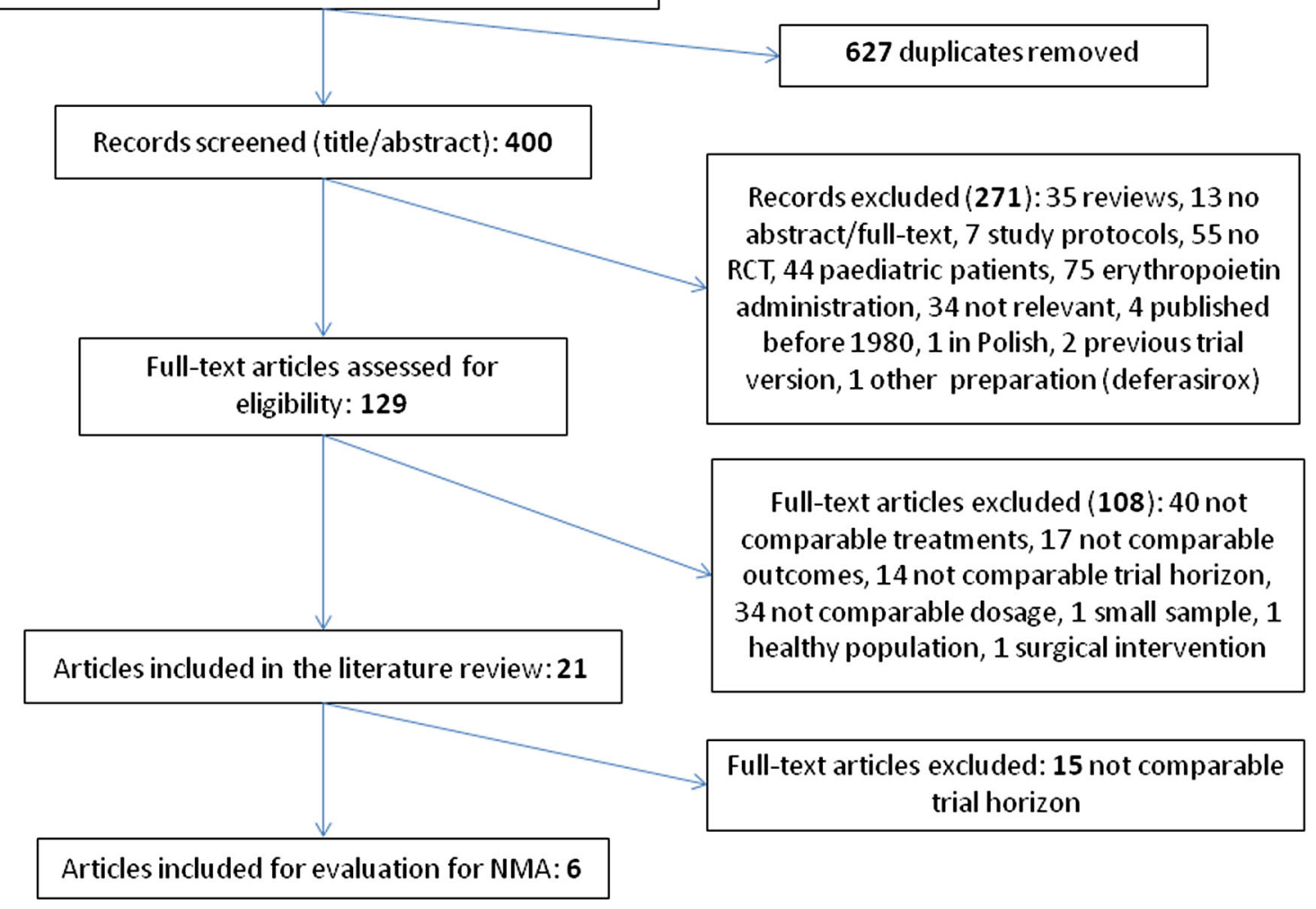

Fig. 1 Study selection process 


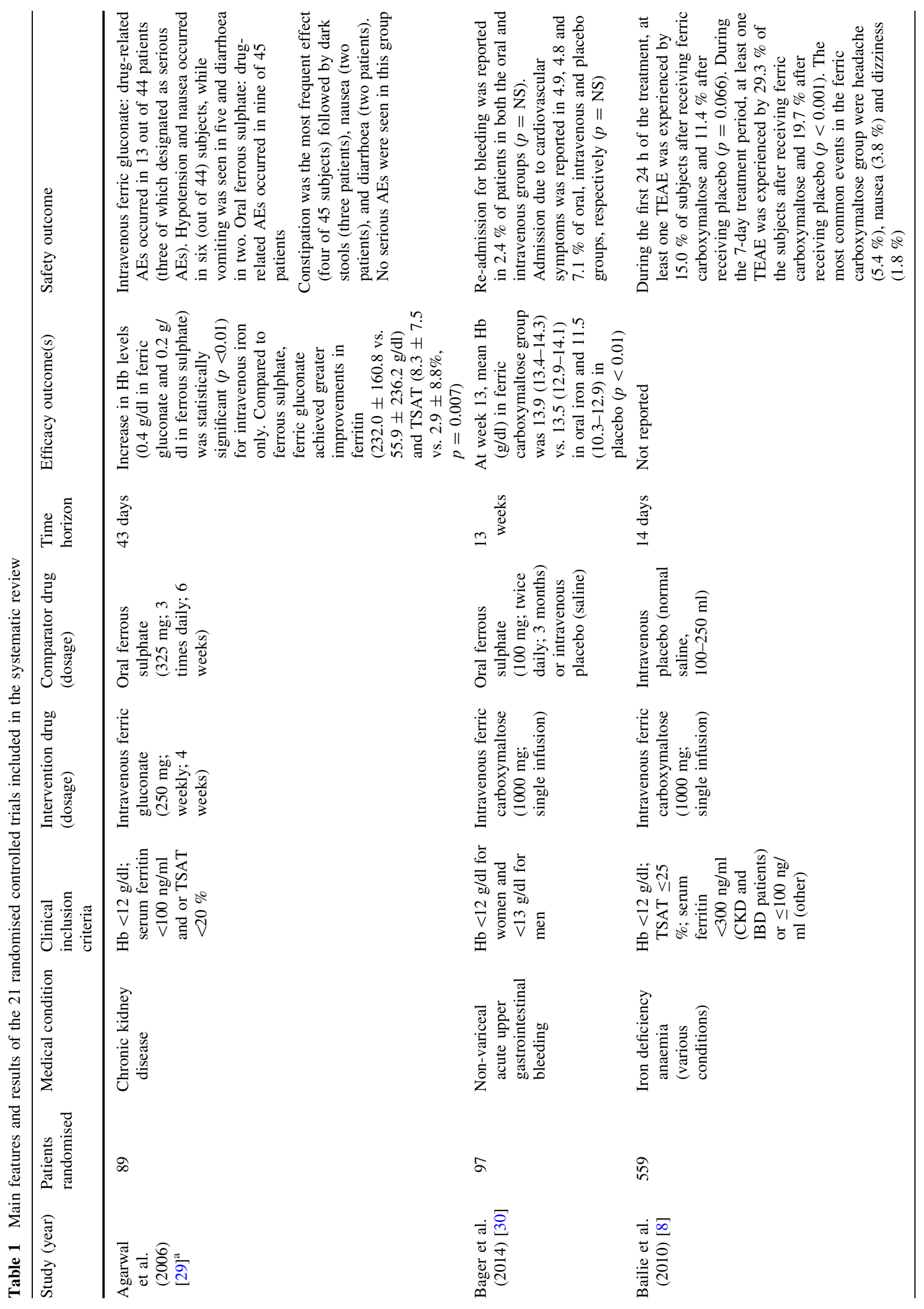




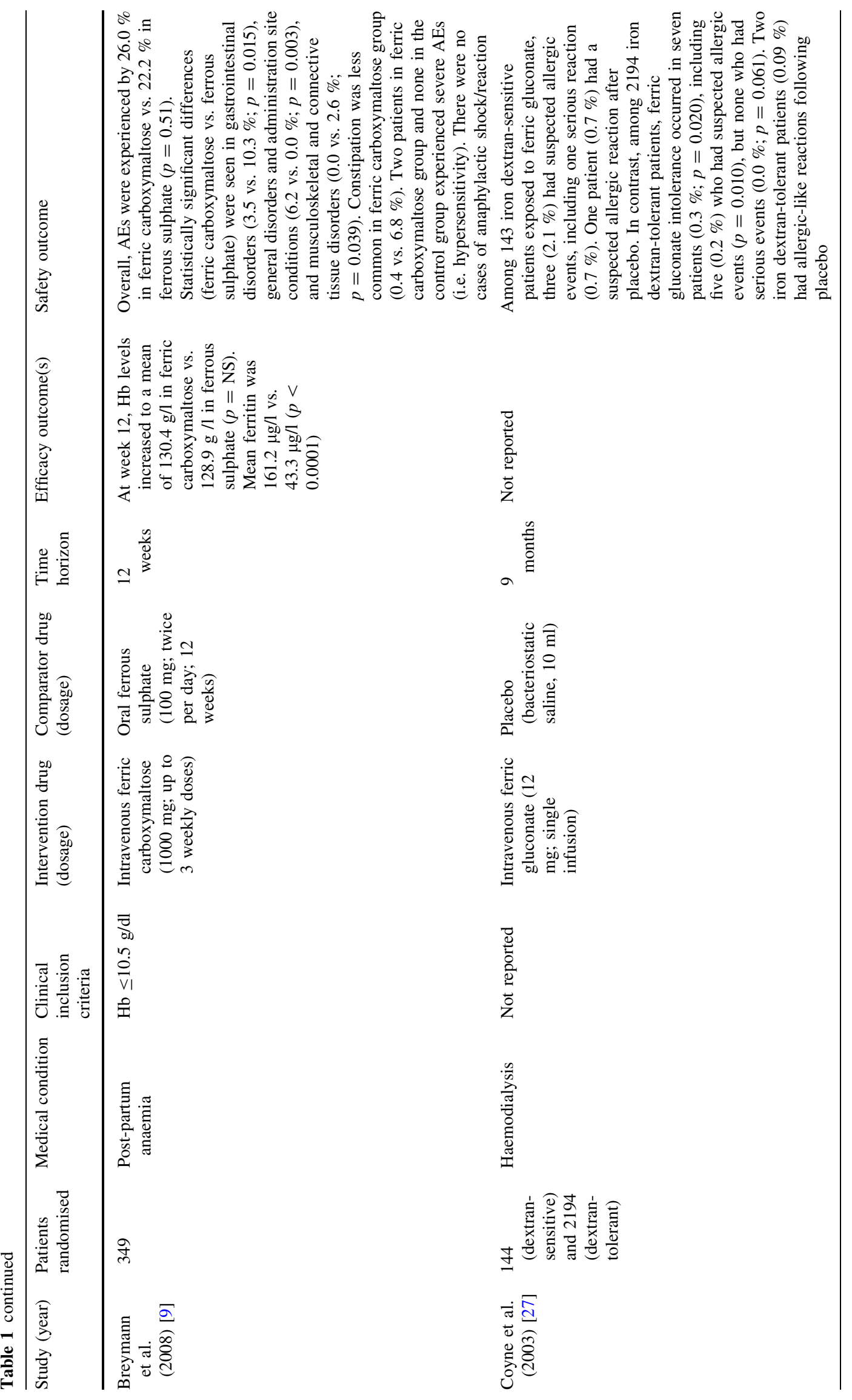




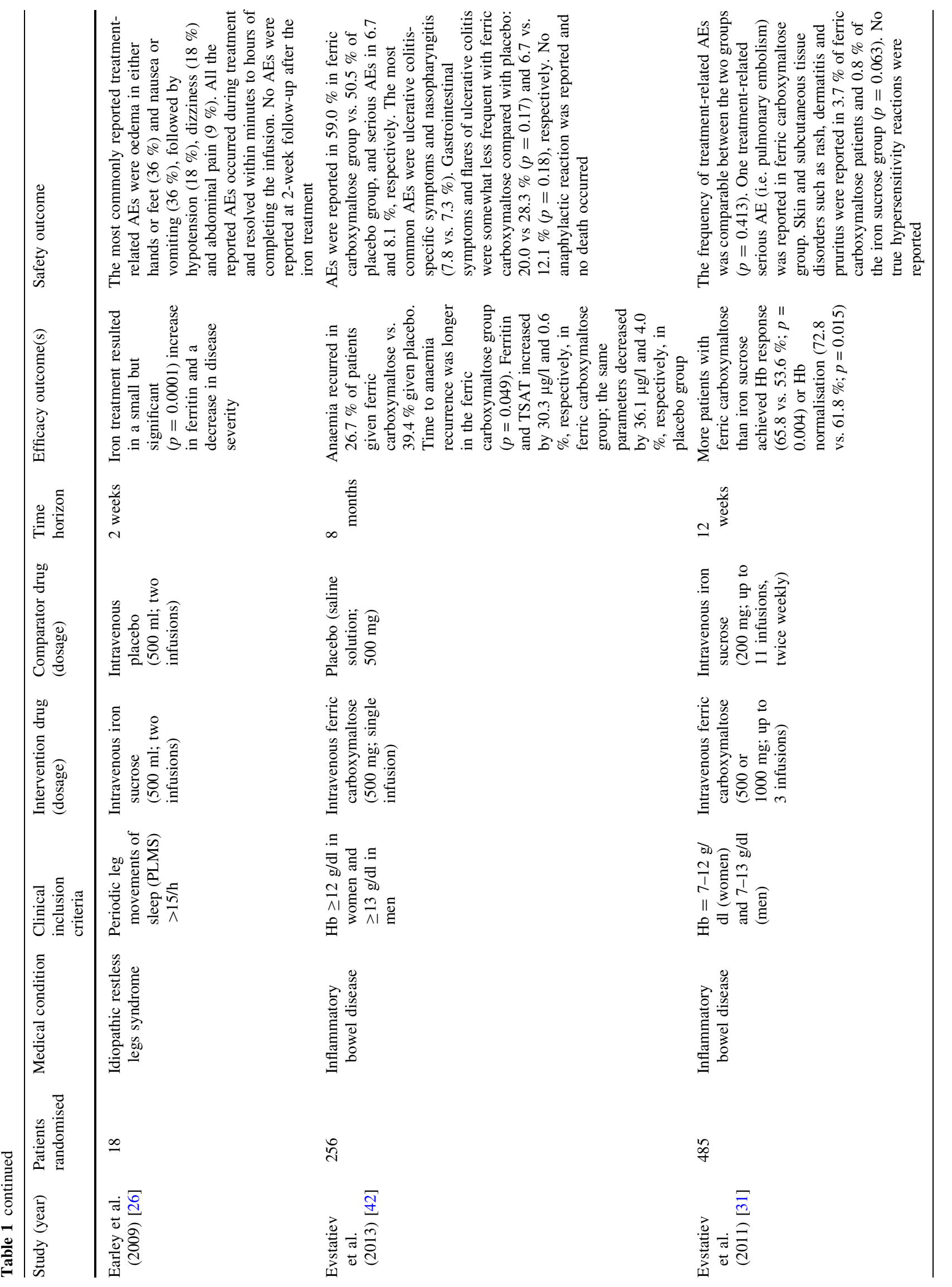




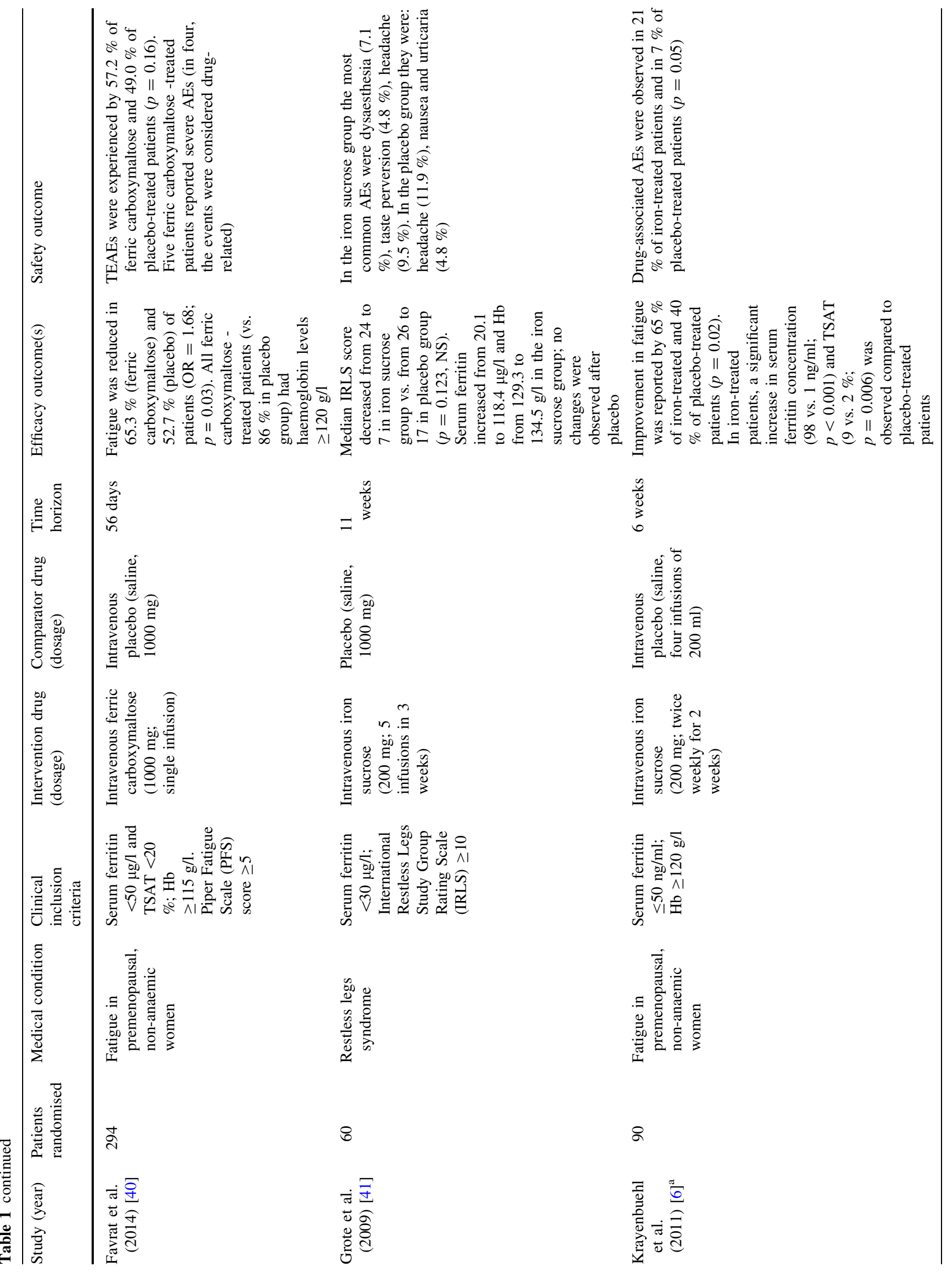




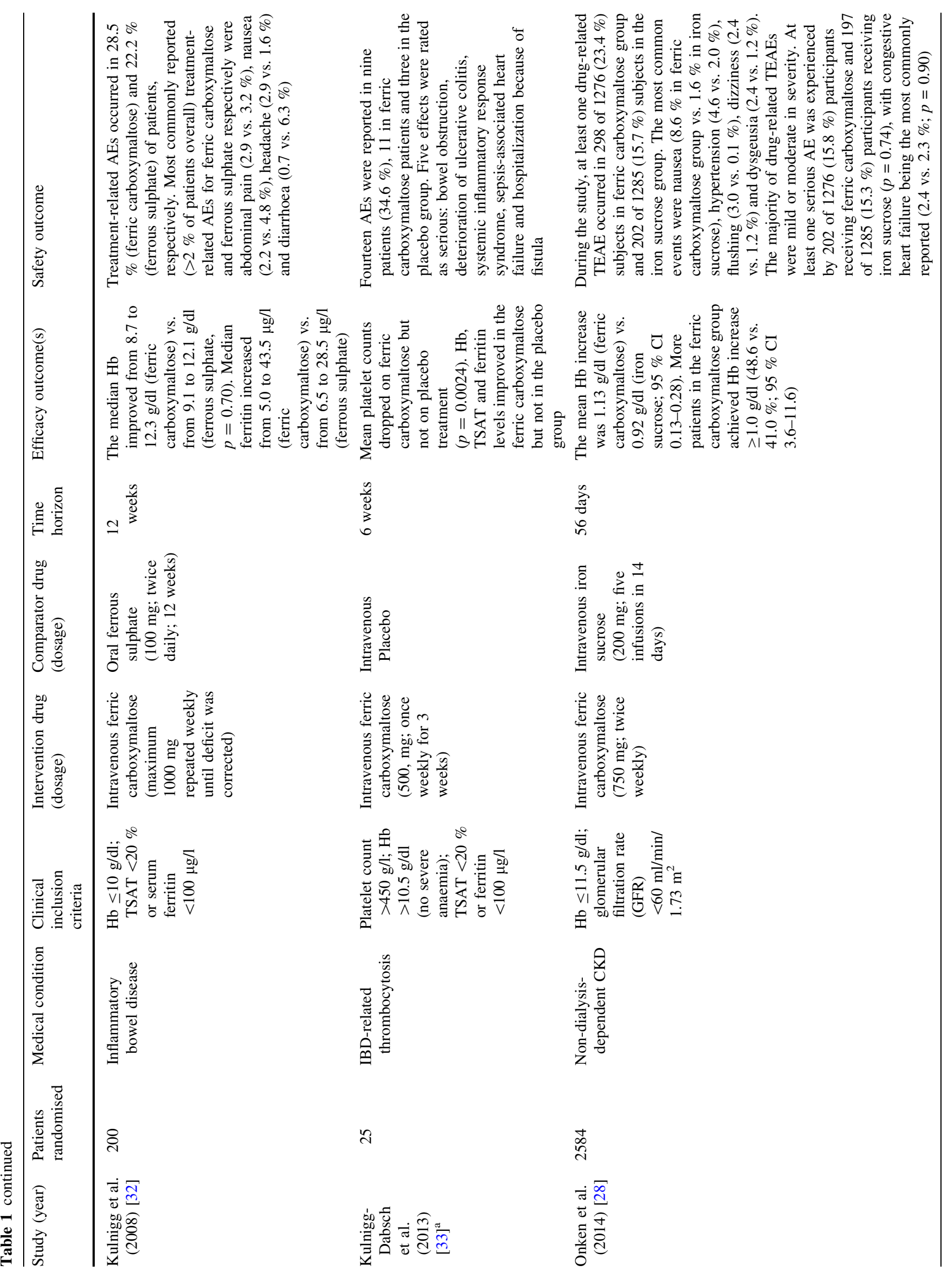




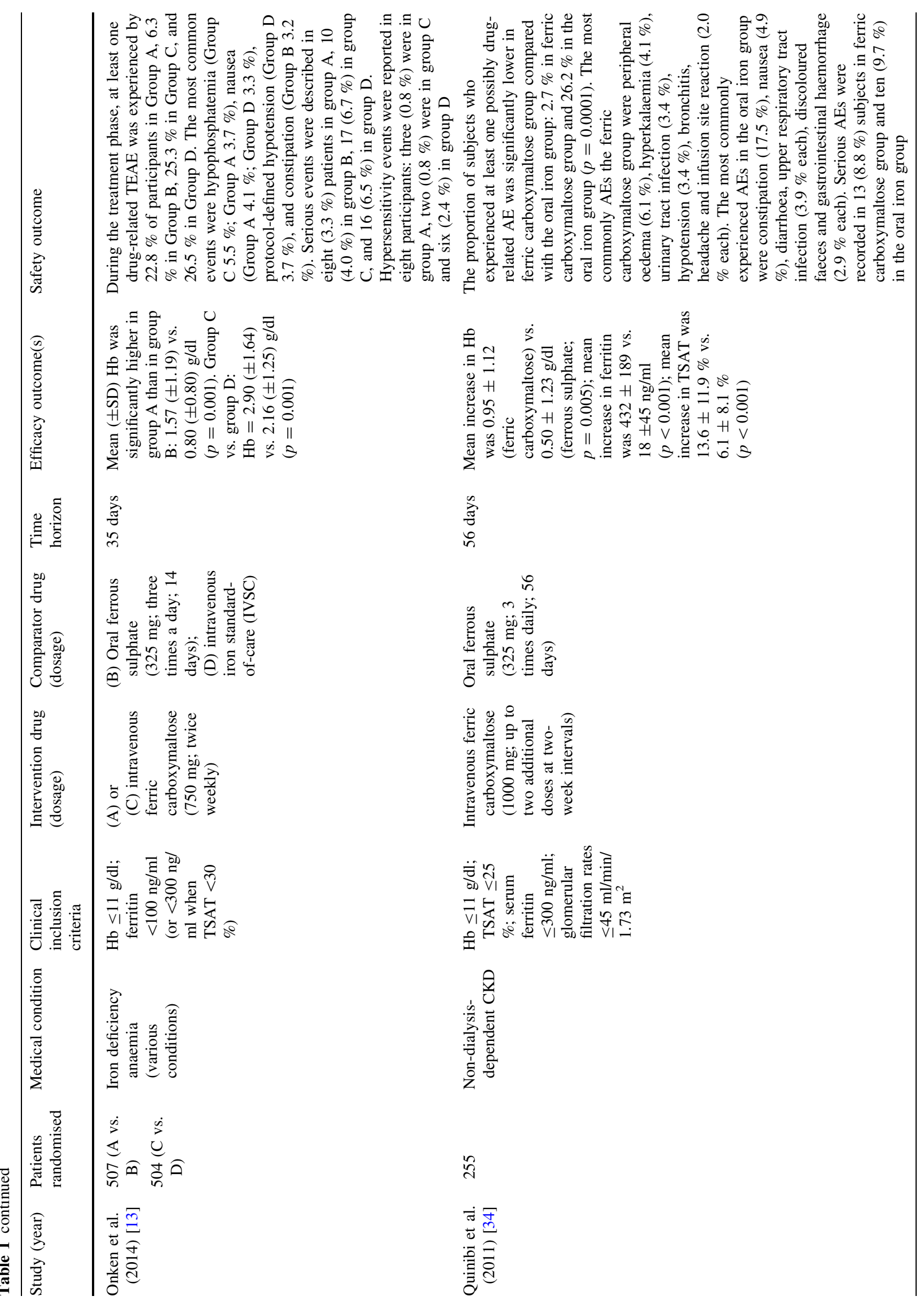




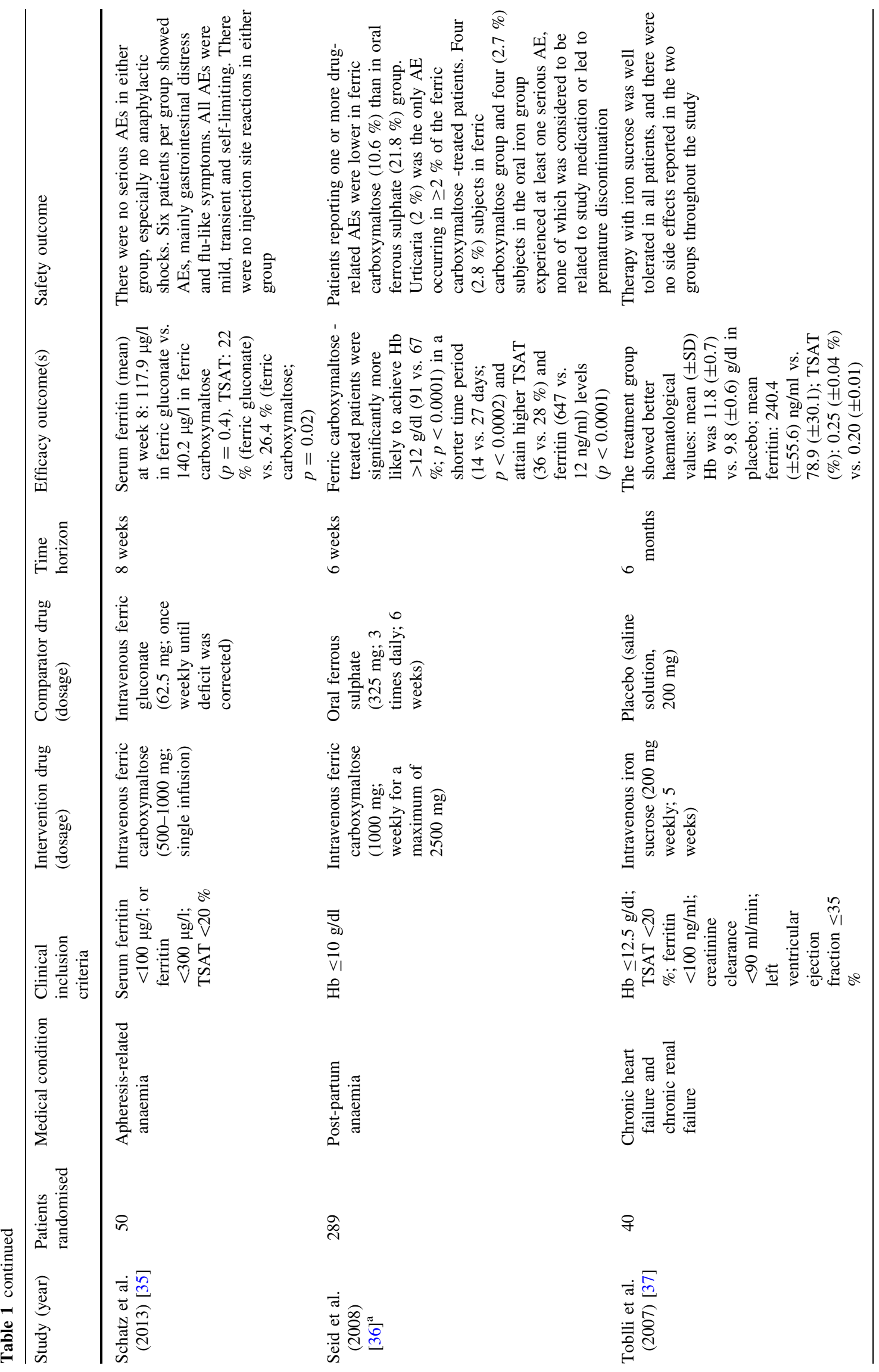




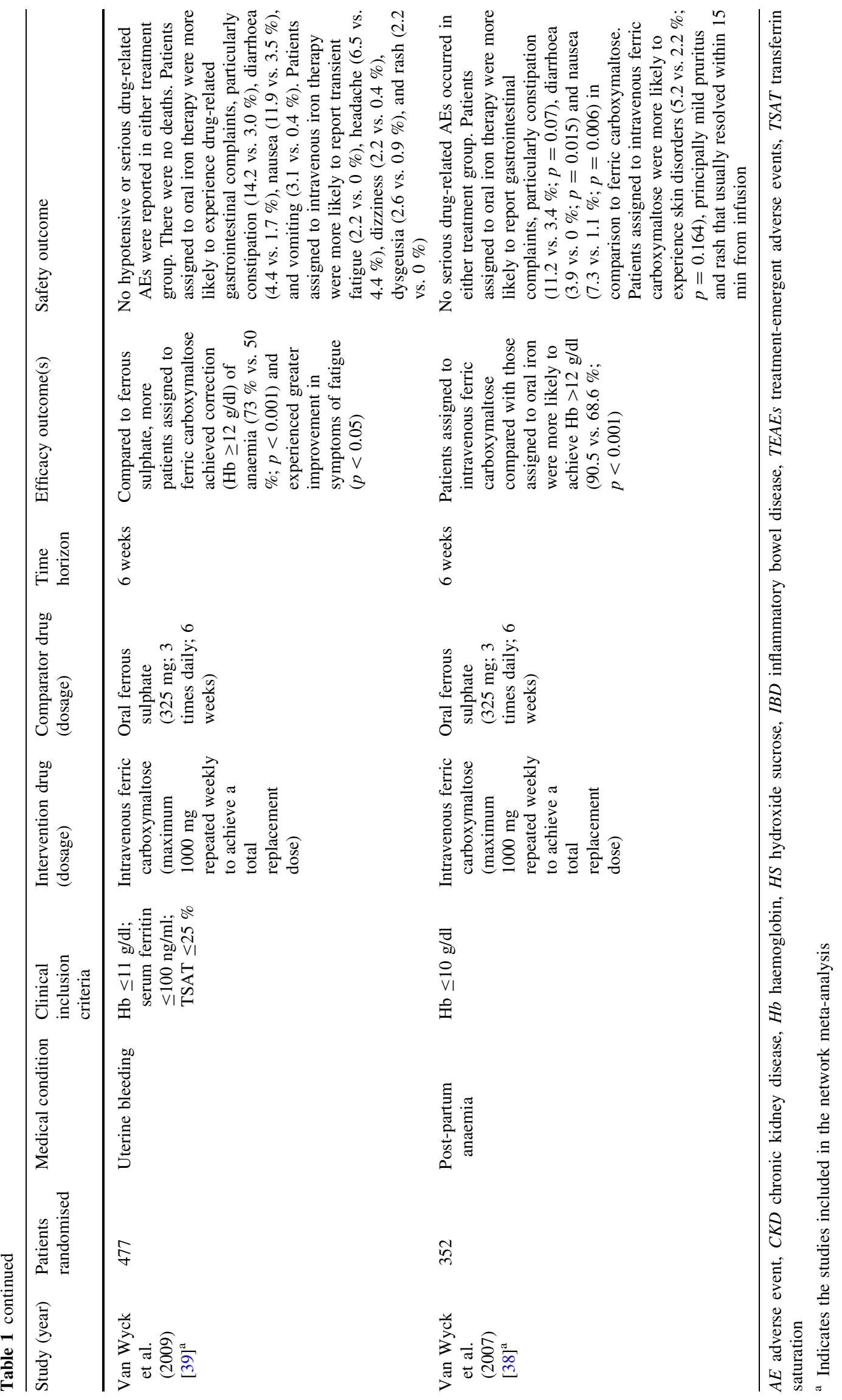


Eight trials [9, 12, 30, 32, 34, 36, 38, 39] compared intravenous ferric carboxymaltose to ferrous sulphate (oral iron) and in one of these studies [13] ferric carboxymaltose was also compared to intravenous standard of care in patients intolerant to oral iron. Since the formulation constituting the 'standard of care' was not specified in the article, this comparison was excluded from the analysis. In another trial [30], an additional comparison was performed between ferric carboxymaltose and intravenous placebo. In four articles [8, 33, 40, 42], ferric carboxymaltose was compared to placebo, while in two $[28,31]$ it was compared to iron sucrose. In two RCTs [27, 29], ferric gluconate was assessed against oral iron (ferrous sulphate) and placebo, respectively; in four studies [6, 26, 37, 41], iron sucrose was compared to placebo. Finally, one study only directly compared ferric carboxymaltose to ferric gluconate [35]. Thus, the overall number of comparisons $(n=22)$ exceeded the number of studies $(n=21)$ due to a study [30] investigating more than two formulations (Fig. 2).

\subsection{Efficacy Outcomes}

In ten studies comparing any iron formulation versus placebo [6, 8, 26, 27, 30, 33, 37, 40-42], efficacy outcomes were always superior in the treatment group. The only study [35] directly comparing ferric carboxymaltose to ferric gluconate revealed that both iron formulations were safe and effective (only six patients per group experienced non-serious AEs), but the increase in haematological parameters was more substantial and rapid in ferric carboxymaltose. When compared to other iron formulations [9, 13, 28, 30, 31, 34, 36, 38, 39], ferric carboxymaltose performed better in the achievement of a rapid and consistent $\mathrm{Hb}$ response with the exception of one study [32], where the difference in median $\mathrm{Hb}$ between ferric carboxymaltose and oral ferrous sulphate was not statistically significant. A higher increase in serum ferritin and TSAT

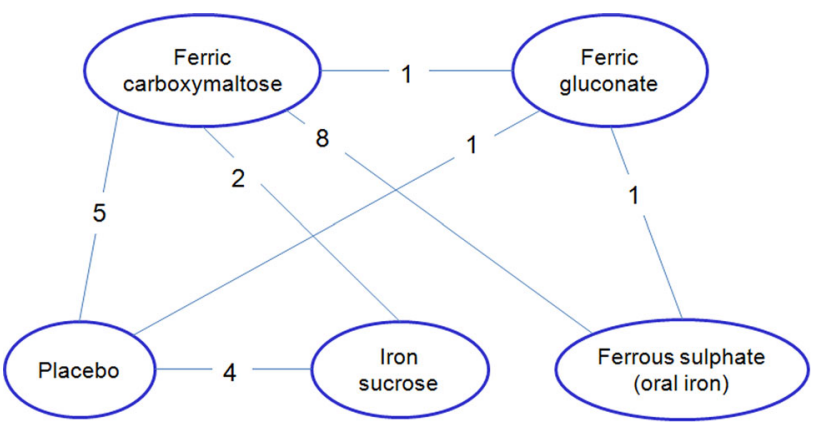

Fig. 2 Schematic representation of comparisons $(n=22)$ among different iron formulations addressed in the included studies $(n=21)$ levels was also observed in patients receiving ferric carboxymaltose compared to other therapies. The only study assessing the performance of ferric gluconate versus oral ferrous sulphate [29] revealed greater improvements in $\mathrm{Hb}$, serum ferritin and TSAT levels in ferric gluconate-treated chronic kidney disease patients than in the oral iron group.

\subsection{Safety Outcomes}

Overall, ferric carboxymaltose was well tolerated and associated with a minimal risk of AEs, even if some authors [8, 9, 32] reported that AEs occurred in a larger proportion of ferric carboxymaltose-treated patients than those receiving oral iron or placebo; the difference seldom reached the significance level $(p<0.05)$. Moreover, some studies $[9,32,34,36,38,39,42]$ revealed that patients who were administered ferric carboxymaltose experienced less drug-related gastrointestinal disorders (e.g. constipation, diarrhoea, nausea and vomiting) than those treated with oral ferrous sulphate. At the same time, patients treated with ferric carboxymaltose iron were more likely to develop skin disorders (e.g. rash, dermatitis and pruritus) that usually resolved within a few minutes of the infusion [9, 31, 34, 38]. Other frequent AEs associated with ferric carboxymaltose administration were fatigue, headache and dizziness [8, 32, 34, 39]. No true cases of anaphylactic reactions were reported [9, 31, 35, 42] and no deaths occurred [39, 42] in patients treated with ferric carboxymaltose.

With reference to other intravenous formulations, the safety profile of ferric gluconate seemed to be favourable [29, 35], with few AEs reported after drug injection. Similarly, iron sucrose was well tolerated and symptoms associated with its administration were mild and resolved rapidly [26, 28, 31, 37].

\subsection{Risk of Bias Assessment}

The risk of bias assessment for the included studies is presented in Figs. 3 and 4. Eight studies [9, 13, 29, 31, 34, 36, 38, 39] were open-label RCTs without blinding of patients and personnel. In two cases [28, 35] no indication on blinding was reported while in two other cases [40, 42] blinding was performed for patients only. For the latter, in one case the authors stated that the primary end-point $(\mathrm{Hb}$ values) was unlikely to be affected by the study personnel's awareness of the treatment. All the remaining studies were double-blind RCTs.

In seven cases the sequence generation process was not described [8, 9, 13, 26, 27, 34, 35], while all the other studies showed low selection biases. 


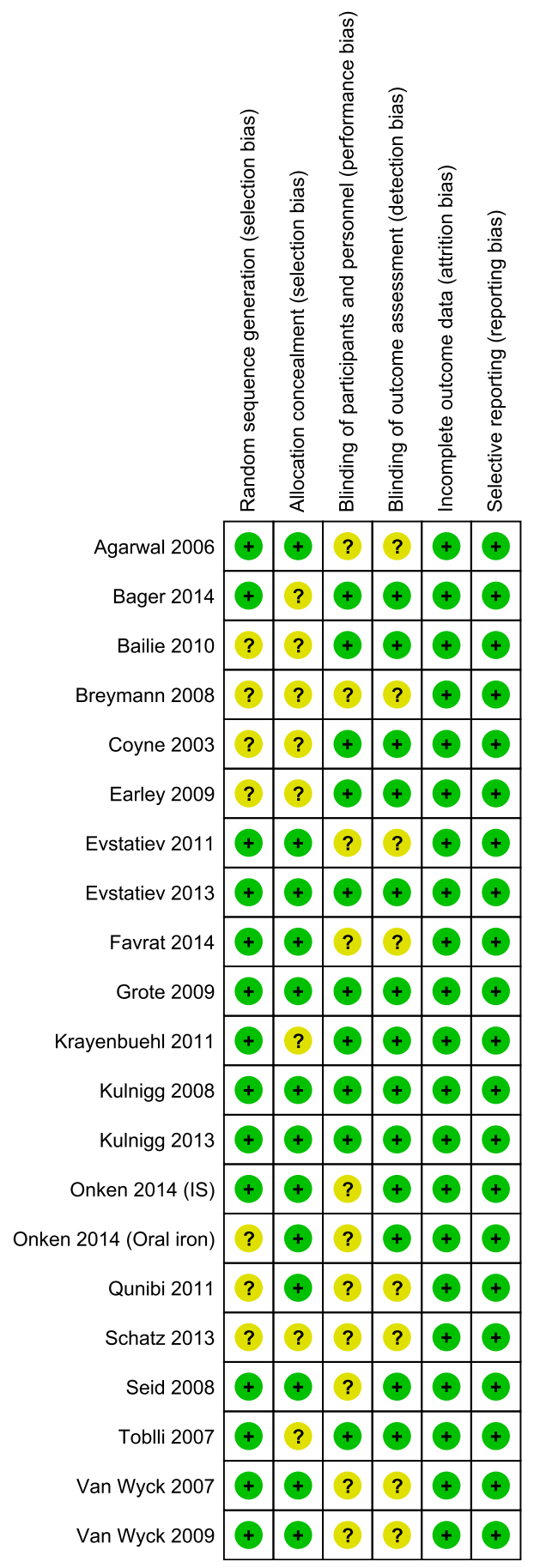

Fig. 3 Risk of bias summary: judgements regarding risks of bias for each study included in the systematic review $(n=21)$. The symbol ' + ' represents low risk of bias, while the symbol '?' represents unclear risk of bias

\subsection{Network Meta-analysis}

Due to the high heterogeneity in trials included in the systematic review, the NMA was limited to those adopting a time horizon of 6 weeks (or 43 days), which was the most frequent period reported. Six [6, 29, 33, 36, 38, 39] out of 21 studies were included in the NMA (see Table 1, studies with "a" label). Studies reported a mean iron dose in the range $800-1600 \mathrm{mg}$ for intravenous formulations, while a daily dose of $975 \mathrm{mg}$ (i.e. $325 \mathrm{mg}$ three times daily for 6 weeks) was administered for oral comparators.

The analyses focused on the increase of $\mathrm{Hb}$ and serum ferritin levels after iron administration. In details, the differences between final and initial values of these parameters when using ferric carboxymaltose were compared to the differences obtained with the other iron formulations; the mean difference of these differences ('delta') was estimated for each couple of treatments (i.e. ferric carboxymaltose vs. competitors) across the studies.

The network of studies considered is reported in Fig. 5, where numbers indicate how many comparisons were performed across the different formulations. The best performing networks were considered: random effects model (Deviance Information Criterion: 55.86 for random effects model vs. 74.69 for fixed effects model) for the serum ferritin NMA and the fixed effects model (Deviance Information Criterion: 5.50 for fixed effects model vs. 5.91 for random effects model) for the $\mathrm{Hb}$ NMA.

As regards serum ferritin $(\mu \mathrm{g} / \mathrm{l})$, the mean difference over the study period (final value - basal value) was significantly larger for ferric carboxymaltose compared to oral iron (delta 172.76; 95 \% CI 66.7-234.4) (see Fig. 6).

Ferric carboxymaltose was superior in comparison to placebo and ferric gluconate with a delta of $65.2(95 \% \mathrm{CI}$ -66.5 to 192.5 ) and 1.5 (95\% CI -131.4 to 122.8 ), respectively. Iron sucrose was superior to ferric carboxymaltose with a delta of 21.4 (95\% CI -160.7 to 118.4). None of the comparisons was statistically significant.

As regards $\mathrm{Hb}(\mathrm{g} / \mathrm{dl})$, the mean difference over the study period (final value - basal value) was significantly larger for ferric carboxymaltose compared to ferric gluconate (delta 0.6; $95 \%$ CI 0.2-0.9), placebo (delta 2.1; $95 \%$ CI 1.2-3.0) and oral iron (delta 0.8; $95 \%$ CI 0.6-0.9) (see Fig. 7). Ferric carboxymaltose was superior to iron sucrose with a delta of 1.1 (95\% CI -1.8 to 3.9) but without statistical significance.

\section{Discussion}

Iron deficiency is a common nutritional deficit, affecting men and women of all ages, races and ethnic groups. In some cases, iron stores may be depleted so as to lead to anaemia (IDA), a severe condition associated with a number of chronic diseases (mainly of the kidney and the bowel) and particular events in women's lives (such as pregnancy and significant menstrual blood loss in young 
Fig. 4 Risk of bias graph: judgements regarding risks of bias presented as percentages across all studies included in the systematic review $(n=21)$. This figure illustrates, for each considered bias domain, the proportion of studies falling in each category of risk (low risk of bias, high risk of bias, unclear risk of bias)

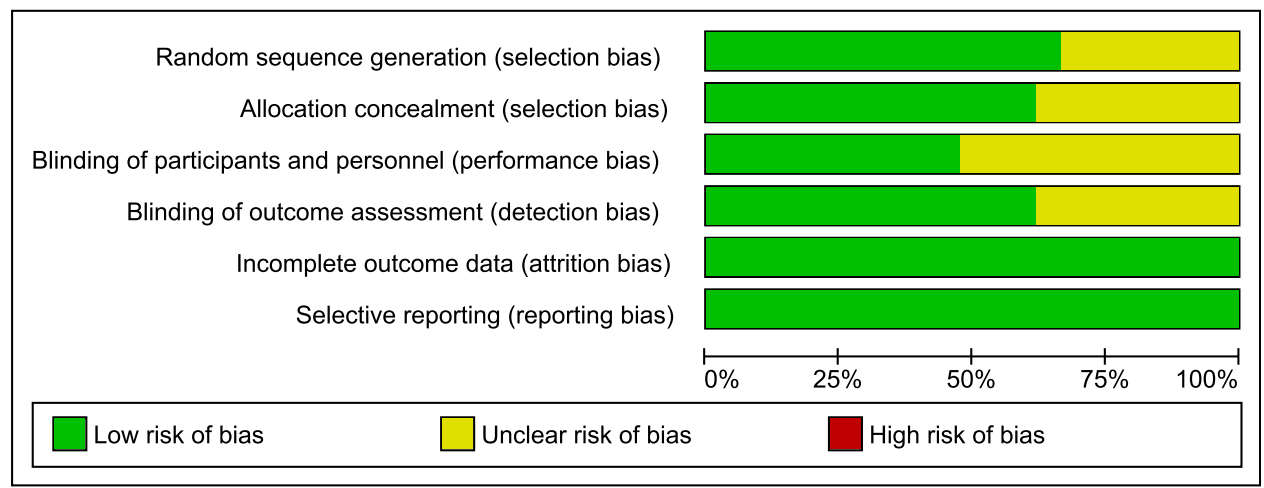

Mean difference in haemoglobin

(each treatment vs. ferric carboxymaltose)

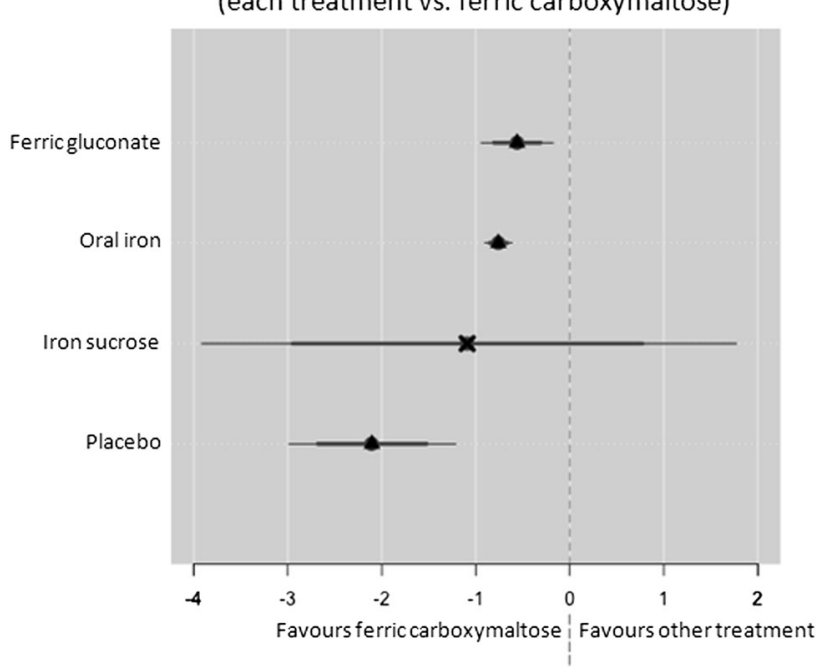

Fig. 7 Network meta-analysis (NMA) results on haemoglobin (Hb). Central dots represent posterior medians, triangles and crosses represent posterior means; thin lines are $95 \%$ credible intervals, while thicker ones are $80 \%$ credible intervals; a triangle indicates that ferric carboxymaltose is significantly superior

effectiveness, safety and cost; however, this formulation presents a number of disadvantages, such as low absorption of iron and high incidence of gastrointestinal side effects. Moreover, iron stores are replenished most effectively and rapidly when intravenous iron supplementation is administered; therefore, parenteral iron administration was introduced in clinical practice to overcome limitations and risks related to oral iron.

Currently, different iron formulations for intravenous infusion are available. These products are quite similar in terms of safety profile but differ in the content and frequency of the doses administered. The present study aimed at improving the level of evidence to support the indication of the available intravenous iron formulations and, particularly, of the most recent preparation, ferric carboxymaltose, brought into clinical practice. women). Patients suffering from IDA and other ID-related conditions can benefit from iron therapy. Oral iron is the first-line treatment for most patients due to its 
Twenty-one RCTs involving ferric carboxymaltose, iron sucrose and ferric gluconate were analysed in order to retrieve information on clinical efficacy and safety of the different iron formulations. Ferric carboxymaltose treatment was shown to be superior in improving IDA compared to other iron regimens. Ferric carboxymaltose superiority is justified by the large amount of iron that can be administered through a small number of injections; this leads to quicker iron replacements in the body and consequently a higher success in anaemia correction. A ferric carboxymaltose dosing regimen was also well tolerated, with a low number of AEs reported, most minor.

The present systematic literature review mainly confirms the results already published by other authors [4345]. The early response obtained with ferric carboxymaltose (from day 7 onwards) implies a substantial improvement in time-to-response and patient convenience and emphasises the clinical relevance of this treatment. From the Patient Blood Management (PBM) perspective [46] that aims at optimising the care of patients who might need blood transfusions, ferric carboxymaltose could represent an effective alternative to transfusions in pre-operative settings or in cases of severe anaemia $(\mathrm{Hb}<8 \mathrm{~g} / \mathrm{dl})$, while in patients with non-dialysis-dependent renal failure, ferric carboxymaltose administration might avoid the use of erythropoietin-stimulating agents. In this way, PBM can improve patient outcomes and reduce healthcare costs, while ensuring that blood components are available for the patients who need them.

Due to the paucity of RCTs comparing ferric carboxymaltose with other parenteral iron formulations, a NMA was performed to combine the highest quality (direct and indirect) evidence regarding the efficacy of the novel formulation. The analyses focused on the increase of $\mathrm{Hb}$ and serum ferritin levels after the administration of the iron formulations considering a time horizon of 6 weeks. Mean differences in serum ferritin $(\mu \mathrm{g} / \mathrm{l})$ were significantly larger for ferric carboxymaltose compared to oral iron, and in $\mathrm{Hb}$ $(\mathrm{g} / \mathrm{dl})$ compared to oral iron, ferric gluconate and placebo. Although ferric carboxymaltose superiority over oral iron and placebo was already demonstrated in several trials [13, 30, 33, 34, 36, 38-40, 42], NMA results supported these findings comparing ferric carboxymaltose also to ferric gluconate in terms of improvements in serum ferritin levels. At present, only one trial directly compared ferric carboxymaltose to ferric gluconate [35]; this study reported a significant difference in serum ferritin levels achieved by the two preparations but over a limited period of treatment (i.e. 4 weeks).

Further RCTs are needed to establish the role of ferric carboxymaltose with respect to the other intravenous formulations in patients with IDA. These studies are essential to provide more direct evidence of the comparisons and should also focus on a short time horizon (i.e. 2 weeks) to highlight the formulations suitable to avoid blood transfusions in pre-operative settings. In the current review, only two studies $[32,36]$ reported high haematological responses for ferric carboxymaltose at 2 weeks, but both considered placebo as the comparator.

The present study was based on an extensive bibliographical search that entailed the inclusion of all published clinical trials addressing different intravenous formulations considering any ID-related medical condition. However, this review presents a number of limitations, including those typical of systematic searches and indirect comparisons of interventions. First, a selection bias may have occurred, since only four databases were searched and 'grey' literature such as unpublished studies and trial registers were omitted; the existence of some publication biases cannot be excluded either. Secondly, clinical outcomes, time horizon and treatment dose were not consistent across the studies and a quantitative synthesis of results was possible for six studies only. Moreover, $\mathrm{Hb}$ levels for inclusion criteria and for the assessment of the primary outcome varied a lot in the studies; in some studies, only severe anaemic patients $(\mathrm{Hb}<10.5 \mathrm{~g} / \mathrm{dl})$ were recruited, while in others these cases were excluded on purpose. In some articles, the primary outcome was expressed in terms of difference in $\mathrm{Hb}$ levels $(\mathrm{g} / \mathrm{dl})$, while in others authors reported the percentage of patients who recovered from IDA, thus performing a comparison of treatment effects was complicated.

In the included studies, no health-related quality-of-life and economic data were available, thus a comparison in terms of cost effectiveness of the different intravenous iron formulations was not feasible. Anyway, some authors showed the cost effectiveness of ferric carboxymaltose compared to placebo in patients with chronic heart failure [47-50], while others showed that treatment with ferric carboxymaltose also improved the quality of life of anaemic patients in the postpartum [38] or in patients with IDA associated with inflammatory bowel disease [32] or heavy uterine bleeding [51]. Moreover, a recent study conducted from an Italian perspective showed that ferric carboxymaltose, due to the low number of infusions needed, might be a cost-saving option from both national health system and hospital points of view when compared to ferric gluconate in the treatment of iron-deficient patients [52].

A systematic literature review also considering cost-effectiveness outcomes would be desirable to give a broader perspective to the analyses.

Despite its appeal in synthesizing all the available evidence for a treatment, NMA clearly has some limitations as well. The most critical one is the potentially high heterogeneity of the studies included in the analysis, both with regard to the reference study populations and to the 
treatment allocation schedule. This may raise some doubts about the validity of the findings. However, the present analysis incorporates all the currently available evidence about iron treatments for IDA and, to the best of our knowledge, this is the first attempt to systematically and quantitatively review the literature in this field.

\section{Conclusions}

Among the different iron formulations available for the treatment of IDA, intravenous ferric carboxymaltose was shown to be superior when compared with other iron regimens. This new formulation can rapidly improve haemoglobin levels and re-establish depleted iron stores in different populations of patients with ID (or IDA) related to a variety of medical conditions (i.e. chronic kidney disease, inflammatory bowel disease, heavy uterine bleeding or postpartum). Moreover, ferric carboxymaltose resulted in a higher increase of serum ferritin levels in comparison to ferric gluconate and showed a high safety profile.

From the Patient Blood Management perspective, ferric carboxymaltose may avoid the use of blood transfusions or other drugs (e.g. erythropoietin-stimulating agents) in patients requiring an iron replacement.

The advantages of ferric carboxymaltose should be further investigated through broader analyses that also include quality-of-life measures and economic outcomes.

\section{Compliance with Ethical Standards}

Funding The present study was funded by Vifor Pharma Italia Srl through an unrestricted grant to CERGAS, Bocconi University, Via Roentgen 1, 20136 Milan, Italy. The authors were solely responsible for carrying out the research project and in writing the manuscript.

Conflict of interest Dr. Melania Marmifero received a fee from Vifor Pharma Italia Srl to participate in the drafting of this manuscript. None of the other authors have any conflicts of interest to declare.

Open Access This article is distributed under the terms of the Creative Commons Attribution-NonCommercial 4.0 International License (http://creativecommons.org/licenses/by-nc/4.0/), which permits any noncommercial use, distribution, and reproduction in any medium, provided you give appropriate credit to the original author(s) and the source, provide a link to the Creative Commons license, and indicate if changes were made.

\section{References}

1. McLean E, Cogswell M, Egli I, Wojdyla D, de Benoist B. Worldwide prevalence of anaemia, WHO Vitamin and Mineral Nutrition Information System, 1993-2005. Public Health Nutr. 2009;12(4):444-54.

2. WHO. Haemoglobin concentrations for the diagnosis of anaemia and assessment of severity. Vitamin and Mineral Nutrition
Information System. World Health Organization, Geneva (WHO/ NMH/NHD/MNM/11.1) (2011). http://www.who.int/vmnis/indica tors/haemoglobin/en. Accessed 30th October 2015.

3. Umbreit J. Iron deficiency: a concise review. Am J Hematol. 2005;78(3):225-31.

4. Shander A, Goodnough LT, Javidroozi M, Auerbach M, Carson J, Ershler WB, et al. Iron deficiency anemia: bridging the knowledge and practice gap. Transfus Med Rev. 2014;28(3):156-66.

5. Liu K, Kaffes AJ. Iron deficiency anaemia: a review of diagnosis, investigation and management. Eur $\mathrm{J}$ Gastroenterol Hepatol. 2012;24(2):109-16.

6. Krayenbuehl PA, Battegay E, Breymann C, et al. Intravenous iron for the treatment of fatigue in nonanemic, premenopausal women with low serum ferritinconcentration. Blood. 2011;118(12): 3222-7.

7. Bregman DB, Morris D, Koch TA, He A, Goodnough LT. Hepcidin levels predict nonresponsiveness to oral iron therapy in patients with iron deficiency anemia. Am J Haematol. 2013;88(2):97-101. doi:10.1002/ajh.23354.

8. Bailie GR, Mason NA, Valaoras TG. Safety and tolerability of intravenous ferric carboxymaltose in patients with iron deficiency anemia. Hemodial Int. 2010;14:47-54.

9. Breymann C, Gliga F, Bejenariu C, Strizhova N. Comparative efficacy and safety of intravenous ferric carboxymaltose in the treatment of postpartum iron deficiency anemia. Int J Gynaecol Obstet. 2008;101(1):67-73.

10. Bisbe E, García-Erce JA, Díez-Lobo AI, Muñoz M. Anaemia Working Group España. A multicentre comparative study on the efficacy of intravenous ferric carboxymaltose and iron sucrose for correcting preoperative anaemia in patients undergoing major elective surgery. Br J Anaesth. 2011;107(3):477-8.

11. Muñoz M, García-Erce JA, Cuenca J, Bisbe E, Naveira E, AWGE (Spanish Anaemia Working Group). On the role of iron therapy for reducing allogeneic blood transfusion in orthopaedic surgery. Blood Transfus. 2012;10(1):8-22.

12. Auerbach $M$, Ballard $H$. Clinical use of intravenous iron: administration, efficacy, and safety. Hematol Am Soc Hematol Educ Program. 2010;2010:338-47.

13. Onken JE, Bregman DB, Harrington RA, Morris D, Acs P, Akright $\mathrm{B}$, et al. A multicenter, randomized, active-controlled study to investigate the efficacy and safety of intravenous ferric carboxymaltose in patients with iron deficiency anemia. Transfusion. 2014;54(2):306-15.

14. http://www.products.sanofi-aventis.us/ferrlecit/ferrlecit.pdf. Accessed 30th October 2015.

15. Moore RA, Gaskell H, Rose P, et al. Meta-analysis of efficacy and safety of intravenous ferric carboxymaltose (Ferinject) from clinical trial reports and published trial data. BMC Blood Disord. 2011;11:4.

16. Moher D, et al. Preferred reporting items for systematic reviews and meta-analyses: the PRISMA statement. Int $\mathrm{J}$ Surg. 2010;8(5):336-41.

17. Sutton AJ, Abrams KR, Jones DR, et al. Methods for metaanalysis in medical research. London: Wiley; 2000.

18. Jansen JP, Crawford B, Bergman G, Stam W. Bayesian metaanalysis of multiple treatment comparisons: an introduction to mixed treatment comparisons. Value Health. 2008;11:956-64.

19. Bucher HC, Guyatt GH, Griffith LE, Walter SD. The results of direct and indirect treatment comparisons in meta-analysis of randomized controlled trials. J Clin Epidemiol. 1997;50(6):683-91.

20. Salanti G. Indirect and mixed-treatment comparison, network, or multiple-treatments meta-analysis: many names, many benefits, many concerns for the next generation evidence synthesis tool. Res Synth Methods. 2012;3:80-97.

21. Jansen JP, Fleurence R, Devine B, Itzler R, Barrett A, Hawkins $\mathrm{N}$, et al. Interpreting indirect treatment comparisons and network 
meta-analysis for health-care decision making: report of the ISPOR task force on indirect treatment comparisons good research practices: part 1. Value Health. 2011;14:417-28.

22. Donegan S, Williamson P, Gamble C, Tudur-Smith C. Indirect comparisons: a review of reporting and methodological quality. PLoS One. 2010;5:e11054.

23. Song F, Loke YK, Walsh T, Glenny AM, Eastwood AJ, Altman DG. Methodological problems in the use of indirect comparisons for evaluating healthcare interventions: survey of published systematic reviews. BMJ. 2009;338:b1147.

24. Lu G, Ades AE. Combination of direct and indirect evidence in mixed treatment comparisons. Stat Med. 2004;23:3105-24.

25. Lunn DJ, Thomas A, Best N, Spiegelhalter D. WinBUGS-a Bayesian modelling framework: concepts, structure and extensibility. Stat Comput. 2000;10:325-37.

26. Earley CJ, Horská A, Mohamed MA, Barker PB, Beard JL, Allen RP. A randomized, double-blind, placebo-controlled trial of intravenous iron sucrose in restless legs syndrome. Sleep Med. 2009;10(2):206-11.

27. Coyne DW, Adkinson NF, Nissenson AR, Fishbane S, Agarwal R, Eschbach JW, Michael B, Folkert V, Batlle D, Trout JR, Dahl N, Myirski P, Strobos J, Warnock DG, Ferlecit Investigators. Sodium ferric gluconate complex in hemodialysis patients. II. Adverse reactions in iron dextran-sensitive and dextran-tolerant patients. Kidney Int. 2003;63(1):217-24.

28. Onken JE, Bregman DB, Harrington RA. Ferric carboxymaltose in patients with iron-deficiency anemia and impaired renal function: the REPAIR-IDA trial. Nephrol Dial Transpl. 2014;29:833-42.

29. Agarwal R, Rizkala AR, Bastani B, et al. A randomized controlled trial of oral versus intravenous iron in chronic kidney disease. Am J Nephrol. 2006;26(5):445-54.

30. Bager P, Dahlerup JF. Randomised clinical trial: oral vs. intravenous iron after upper gastrointestinal haemorrhage. A placebocontrolled study. Aliment Pharmacol Ther. 2014;39(2):176-87.

31. Evstatiev R, Marteau P, Iqbal T, et al. FERGIcor: a randomized controlled trial on ferric carboxymaltose for iron deficiency anemia in inflammatory bowel disease. Gastroenterology. 2011;141:846-53.

32. Kulnigg S, Stoinov S, Simanenkov V, et al. A novel intravenous iron formulation for treatment of anemia in inflammatory bowel disease: the ferric carboxymaltose (FERINJECT) randomized controlled trial. Am J Gastroenterol. 2008;103:1182-92.

33. Kulnigg-Dabsch S, Schmid W, Howaldt $S$, et al. Iron deficiency generates secondary thrombocytosis and platelet activation in IBD: the randomized, controlled thromboVIT trial. Inflamm Bowel Dis. 2013;19(8):1609-16.

34. Qunibi WY, Martinez C, Smith M, Benjamin J, Mangione A, Roger SD. A randomized controlled trial comparing intravenous ferric carboxymaltose with oral iron for treatment of iron deficiency anaemia of non-dialysis-dependent chronic kidney disease patients. Nephrol Dial Transplant. 2011;26(5):1599-607.

35. Schatz U, Arneth B, Siegert G, et al. Iron deficiency and its management in patients undergoing lipoprotein apheresis. Comparison of two parenteral iron formulations. Atheroscler Suppl. 2013;14(1):115-22.

36. Seid MH, Derman RJ, Baker JB, Banach W, Goldberg C, Rogers R. Ferric carboxymaltose injection in the treatment of postpartum iron deficiency anemia: a randomized controlled clinical trial. Am J Obstet Gynecol 2008;199(4):435.e1-7.
37. Toblli JE, Lombraña A, Duarte P, Di Gennaro F. Intravenous iron reduces NT-pro-brain natriuretic peptide in anemic patients with chronic heart failure and renal insufficiency. J Am Coll Cardiol. 2007;50(17):1657-65.

38. Van Wyck DB, Martens MG, Seid MH, et al. Intravenous ferric carboxymaltose compared with oral iron in the treatment of postpartum anemia. A randomized controlled trial. Obstet Gynecol. 2007;110:267-78.

39. Van Wyck DB, Mangione A, Morrison J, et al. Large-dose intravenous ferric carboxymaltose injection for iron deficiency anemia in heavy uterine bleeding: a randomized, controlled trial. Transfusion. 2009;49(12):2719-28.

40. Favrat B, Balck K, Breymann C, Hedenus M, Keller T, Mezzacasa A, Gasche C. Evaluation of a single dose of ferric carboxymaltose in fatigued, iron-deficient women-PREFER a randomized, placebo-controlled study. PLoS One. 2014;9(4):e94217.

41. Grote L, Leissner L, Hedner J, Ulfberg J. A randomized, doubleblind, placebo controlled, multi-center study of intravenous iron sucrose and placebo in the treatment of restless legs syndrome. Mov Disord. 2009;24(10):1445-52.

42. Evstatiev R, Alexeeva O, Bokemeyer B, et al. Ferric carboxymaltose prevents recurrence of anemia in patients with inflammatory bowel disease. Clin Gastroenterol Hepatol. 2013;11:269-77.

43. Lyseng-Williamson KA, Keating GM. Ferric carboxymaltose: a review of its use in iron-deficiency anaemia. Drugs. 2009;69(6):739-56.

44. Keating GM. Ferric carboxymaltose: a review of its use in iron deficiency. Drugs. 2015;75(1):101-27.

45. Bregman DB, Goodnough LT. Experience with intravenous ferric carboxymaltose in patients with iron deficiency anemia. Ther Adv Hematol. 2014;5(2):48-60.

46. Shander A, Van Aken H, Colomina MJ, Gombotz H, Hofmann A, Krauspe $\mathrm{R}$, et al. Patient blood management in Europe. Br J Anaesth. 2012;109(1):55-68.

47. Hofmarcher T, Borg S. Cost-effectiveness analysis of ferric carboxymaltose in iron-deficient patients with chronic heart failure in Sweden. J Med Econ. 2015;18(7):492-501.

48. Comín-Colet J, Rubio-Rodríguez D, Rubio-Terrés C, EnjuanesGrau C, Gutzwiller FS, Anker SD, Ponikowski P. A cost-effectiveness analysis of ferric carboxymaltose in patients with iron deficiency and chronic heart failure in Spain. Rev Esp Cardiol (Engl Ed). 2015;68(10):846-51.

49. Gutzwiller FS, Schwenkglenks M, Blank PR, Braunhofer PG, Mori C, Szucs TD, et al. Health economic assessment of ferric carboxymaltose in patients with iron deficiency and chronic heart failure based on the FAIR-HF trial: an analysis for the UK. Eur J Heart Fail. 2012;14(7):782-90.

50. Lim EA, Sohn HS, Lee H, Choi SE. Cost-utility of ferric carboxymaltose $\left(\right.$ Ferinject $^{\circledR}$ ) for iron-deficiency anemia patients with chronic heart failure in South Korea. Cost Eff Resour Alloc. 2014;10(12): 19 .

51. Gordon SS, Hadley PE, Van Wyck DB, et al. Iron carboxymaltose, a new intravenous iron agent for iron deficiency anemia in heavy uterine bleeding. Obstet Gynecol. 2007;109(Suppl 4): $108 \mathrm{~S}$.

52. Rognoni C, Tarricone R, Meregaglia M. Impatto economico dell'utilizzo di carbossimaltosio ferrico in pazienti con anemia da carenza di ferro nelle regioni italiane. MECOSAN. Manag Econ Sanit. 2015;93:99-114. 